\title{
Public Health Considerations among People who Inject Drugs with HIV/HCV Co-Infection: A Review
}

\author{
Rachel M. Murdock · Marisa B. Brizzi · Omar Perez · Melissa E. Badowski
}

Received: October 11, 2018 / Published online: January 3, 2019

(C) The Author(s) 2019

\section{ABSTRACT}

Hepatitis C virus (HCV) and human immunodeficiency virus (HIV) co-infection among persons who inject drugs (PWID) is a major public health concern. There are limited data in clinical trials on the use of direct-acting antiviral (DAA) therapy for treatment of HCV in coinfected PWID. It is critical for these patients to gain access to treatment in order to decrease progression of liver disease and decrease transmission of both HIV and HCV. Additional harm reduction interventions, including needle and syringe programs and opioid substitution treatment, should be made available to this vulnerable population. Despite the importance of DAA treatment, the cost of DAA therapy and access to medical care is still a barrier to appropriate therapy. The purpose of this review is to present available data on the use of DAAs in co-infected PWID, review guideline recommendations for treatment and retreatment of $\mathrm{HCV}$ in co-infected PWID, provide cost considerations for DAA therapy, and provide recommendations

Enhanced digital features To view enhanced digital features for this article go to https://doi.org/10.6084/ m9.figshare.7467554.

R. M. Murdock · M. B. Brizzi · O. Perez ·

M. E. Badowski ( $\square)$

College of Pharmacy, University of Illinois at

Chicago, Chicago, IL, USA

e-mail: badowski@uic.edu about caring for patients who continue to inject drugs.

Keywords: Co-infection; HCV; Hepatitis; HIV/ AIDS; Injection drug use; Public health; PWID

\section{BACKGROUND}

A recent study estimated there were 15.6 million persons who inject drugs (PWID) globally [1]. It was also estimated that 52.3\% (8.2 million people) of current PWID have been exposed to hepatitis $\mathrm{C}$ virus (HCV), while 17.8\% (2.8 million people) of PWID are living with human immunodeficiency virus (HIV). Due to the overlapping routes of parenteral transmission, HIV and HCV infections are a major global public health concern. There were an estimated 2.3 million cases of $\mathrm{HIV} / \mathrm{HCV}$ co-infection in individuals globally, of which 1.4 million (59\%) were also PWID [2]. Eastern Europe and Central Asia have the greatest burden due to the large HIV-infected PWID population of 688,100 , of which an estimated $83 \%$ (567,700 people) of PWID were co-infected with HIV/HCV. North America has an estimated 187,000 patients living with HIV (PLWH) who were also PWID. Of these, 153,300 PWID were co-infected with $\mathrm{HIV} / \mathrm{HCV}$. Additionally, there is evidence to support that, in high-income countries, PWID were the main risk group for HCV transmission, 
contributing to more than $90 \%$ of new infections in settings such as the United Kingdom (UK) [3].

International guidelines recommend prioritizing treatment in individuals at risk of transmitting HCV [4]. Unfortunately, a large number of $\mathrm{HIV} / \mathrm{HCV}$ co-infected PWID remain untreated for HCV $[5,6]$. Identifying the barriers that hinder the implementation of treatment in PWID is crucial to the success of therapy. Importantly, evidence supports the effectiveness of harm reduction interventions, such as needle and syringe programs (NSP) and opioid substitution treatment (OST), in reducing HIV transmission and HCV and HIV incidence [7]. Therefore, it is imperative to identify a treatment system that provides an opportunity for PWID to obtain treatment in order to prevent HIV and HCV transmission and reduce long-term morbidity in PWID. The information in this paper is based on previously conducted studies and does not contain any studies with human participants or animals performed by any of the authors.

\section{GUIDELINE GUIDANCE}

The American Association for the Study of Liver Diseases (AASLD) and the European Association for the Study of the Liver (EASL) do not have specific treatment recommendations in PWID and are co-infected with HIV/HCV $[4,8]$. Directacting antiviral (DAA) therapy continues to be the mainstay of therapy for HCV therapy with or without HIV, yet limited studies exist in PWID. Similar to HCV-monoinfected patients, cure rates are comparable around 95\% sustained virologic response (SVR). However, many studies exclude PWID due to concerns regarding medication and protocol adherence. Active or recent drug use should not be considered a contraindication to HCV therapy, as the DAAs are safe and effective with a reduced length of treatment when compared to interferon-based management. Both guidelines recommend the use of sofosbuvir/velpatasvir or glecaprevir/pibrentasvir to provide pan-genotypic coverage against HCV genotypes 1-6 and can be used in patients who are co-infected. The use of other
DAAs can be considered in this population as long as drug interaction potential is considered in the presence of antiretroviral therapy as well as opioid substitution therapy.

\section{HCV MEDICATION TRIALS IN PEOPLE WITH HIV/HCV CO- INFECTION}

The United States (US) Food and Drug Administration (FDA) had approved ledipasvir/sofosbuvir, glecaprevir/pibrentasvir, sofosbuvir/ velpatasvir, and grazoprevir/elbasvir as singletablet DAAs which have been shown to be safe and effective for use in patients co-infected with $\mathrm{HIV} / \mathrm{HCV}$. Clinical trials supporting the use of these single-tablet DAAs as treatment in the $\mathrm{HIV} / \mathrm{HCV}$ co-infected population are represented in Table 1 with rates of SVR ranging from 95 to $100 \%$, yet specific data in PWID is not reported [9-14]. Each clinical trial included patients co-infected with $\mathrm{HIV} / \mathrm{HCV}$, both on and off antiretroviral therapy (ART) at the time of screening visit, and addressed the handling of data from PWID. ENDURANCE-1 and EXPEDITION-2 both included PWID in the final analysis, and overall rates of SVR for the entire study populations were $100 \%$ and $98 \%$, respectively $[9,10]$. In ENDURANCE-1, 28\% of the total study population reported injection drug use (IDU), while in EXPEDITION-2, 6-9\% of total study population reported IDU within 12 months of screening and $45-63 \%$ reported IDU more than 12 months prior to screening. The National Institute of Allergy and Infectious Diseases (NIAID) ERADICATE study required all opioid-dependent subjects to participate in supervised treatment, with no mention of specifics on urine drug screening (UDS) or pharmacological agents used for OST [11]. Results from NIAID ERADICATE represented SVR rates of $100 \%$ in ART-naïve patients and 97\% in ARTexperienced patients. ION-4 and ASTRAL-5 excluded patients with clinically relevant drug abuse (not defined) or positive drug screen within 12 months of study enrollment $[12,13]$. Interestingly, UDS was only collected at the initial screening visit. C-EDGE CO-INFECTION also excluded patients with clinically relevant 


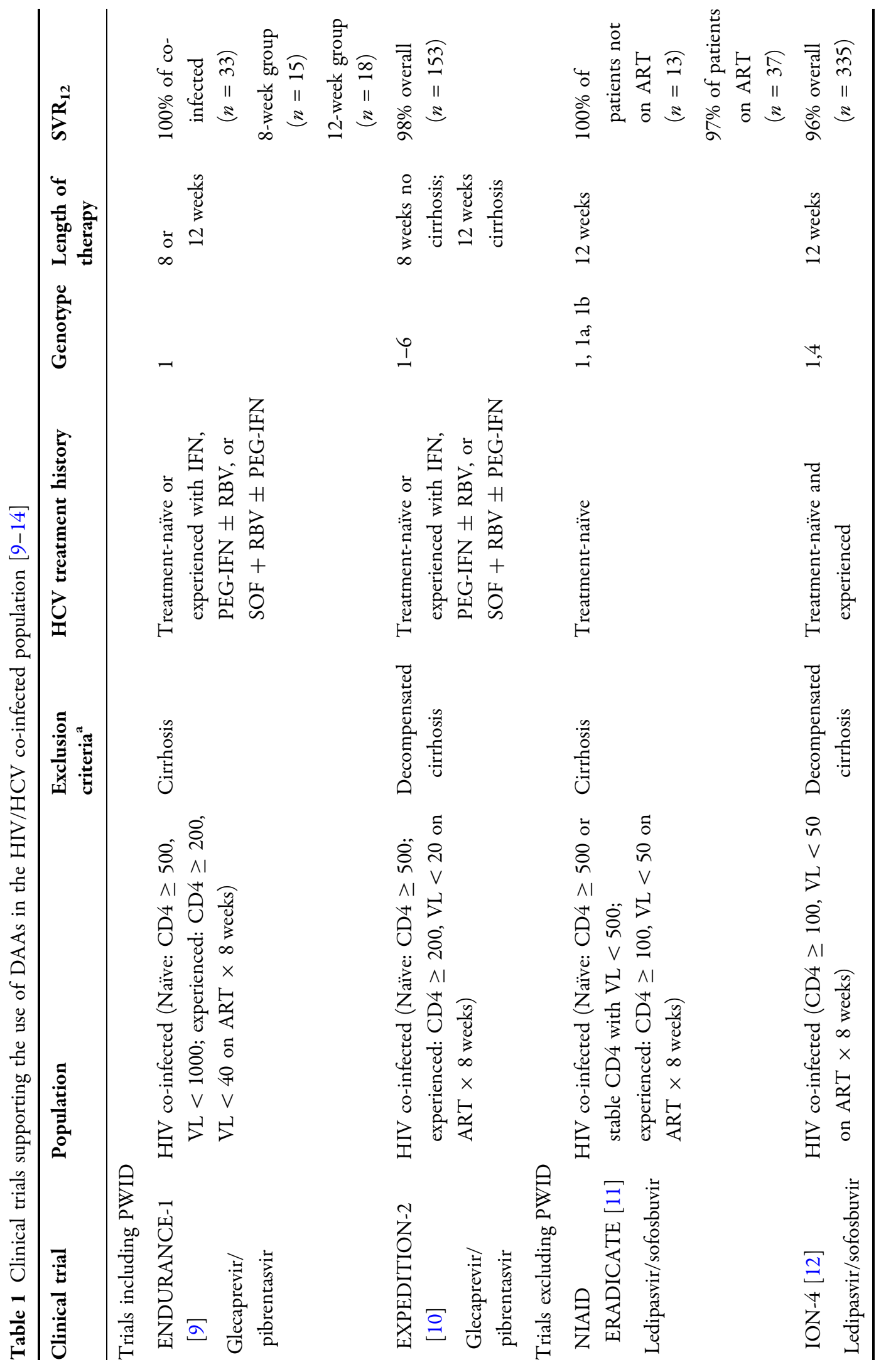




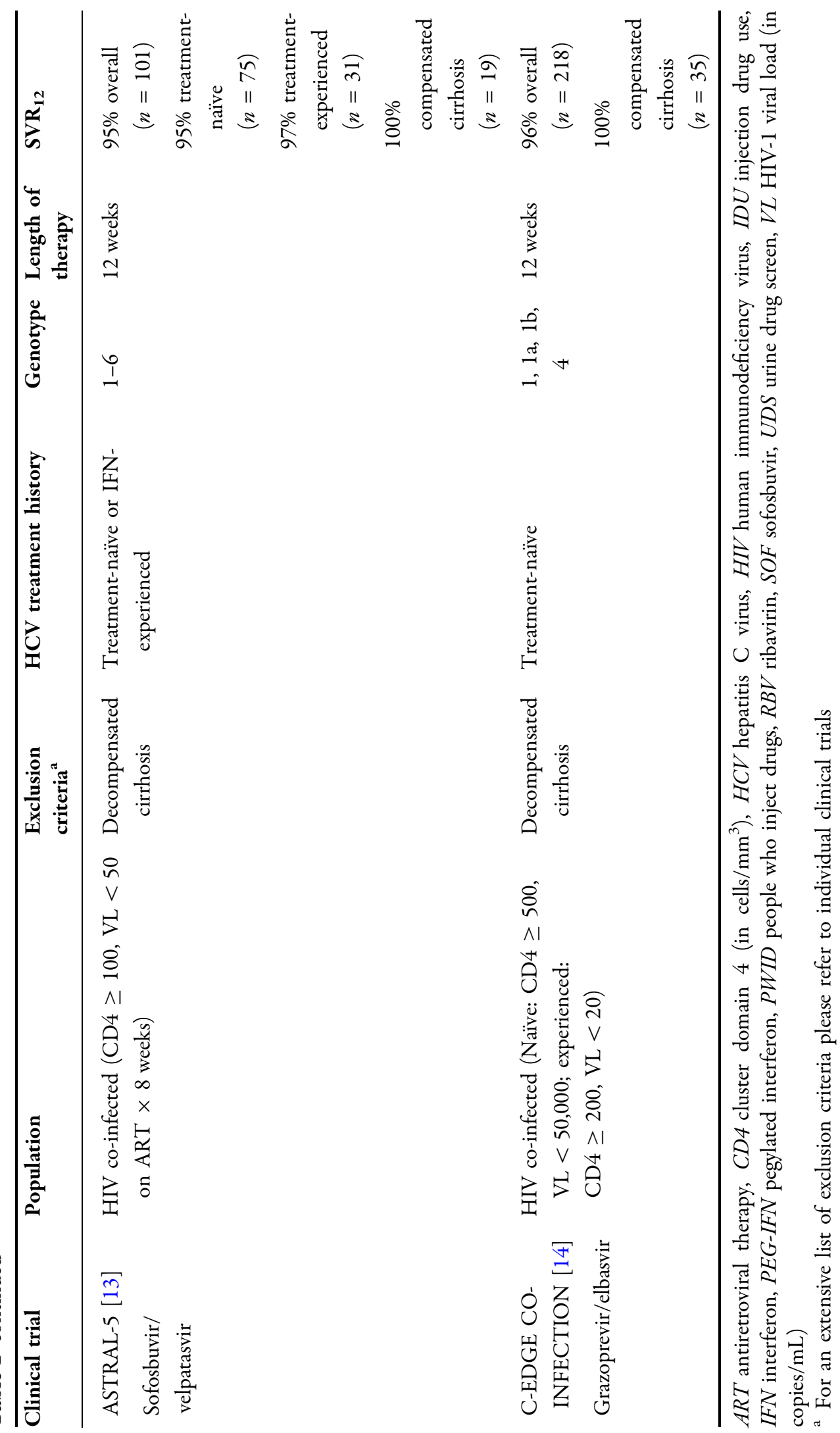


drug abuse (not defined) within 12 months of study enrollment, but did not require UDS at enrollment or any time during the study period [14]. No virologic failure was noted in any of these trials, while NIAID ERADICATE and C-EDGE CO-INFECTION each had patients with transient viremia who later achieved viral suppression with no change in ART $[11,14]$.

Overall, limited studies evaluate the use of DAAs in HIV/HCV co-infected PWID. ENDURANCE-1 and EXPEDITION-2 demonstrated high rates of SVR in PWID using glecaprevir/pibrentasvir for treatment of HCV $[9,10]$. These trials represented the DAA with the most data in the HIV/HCV co-infected PWID population. Despite the limited availability of data, both these studies demonstrate that a HCV cure can be achieved despite all of the considerations and/or concerns regarding active or prior drug use.

\section{COST CONSIDERATIONS}

While the efficacy of the DAA regimens for HCV infection is well established, cost is still a major concern when considering large-scale access to these medications. The sticker price for $\mathrm{HCV}$ treatment is substantially higher than many other medications on the market today. However, the cost-avoidance achieved with these regimens should also be considered. Complications of untreated chronic HCV infection include end-stage liver disease/cirrhosis, hepatocellular carcinoma (HCC), liver transplantation and ultimately death. In 2017, the average total cost for a liver transplant in the United States was US\$812,700. This included, in addition to the surgery, the 30-day pre-transplant work-up, procurement of the organ, hospitalization, post-transplant follow-up, and all medications throughout the process [15]. While mortality associated with hepatocellular carcinoma is decreasing, the overall hospitalization costs have been steadily increasing. One study noted a $\$ 15,153$ increase in the cost per hospitalization between the years 2002 and 2011, after adjusting for inflation [16]. Medical treatment for HCV may decrease the overall economic burden of end-stage liver disease and cirrhosis, which are costly to our healthcare system. Furthermore, the treatment cost also prevents reinfection or further infection of other injection drug users in the PWID population. Even when taking into consideration the high price of medications, the cost-avoidance achieved through treating this patient population rather than delaying treatment has been shown to be cost-effective [17].

The price tag on DAA therapy varies globally, but remains unaffordable to a majority of patients living with HCV [18]. Some low- and medium-income countries have licensing agreements with pharmaceutical companies for tiered pricing of certain DAAs, but these agreements do not include the middle-income countries which suffer the highest burden of $\mathrm{HCV}$ infections. For example, Central and Eastern European countries have the highest costs for DAA therapies, despite having the highest burden of $\mathrm{HIV} / \mathrm{HCV}$ co-infected patients. Low-cost generic DAAs available in India have led to improved patient outcomes and increased cost savings related to $\mathrm{HCV}$ treatment, but the cost of testing and diagnosis still remain high [19]. Generic DAAs are expected to become available in the US in early 2019, which could potentially lead to increased access to treatment and a reduction in the economic burden of HCV [20].

\section{CONSIDERATIONS WHEN CARING FOR PATIENTS WHO CONTINUE TO INJECT DRUGS}

When treating a person who injects drugs with newly diagnosed HIV/HCV co-infection, as with all patients, medication therapy for both infections should be initiated as soon as possible to prevent the progression of liver disease and decrease transmission of the viruses. However, caring for co-infected patients who continue to inject drugs presents health care providers with potential challenges including reinfection, transmission, and nonadherence to antivirals and monitoring programs. Co-infected PWID should be counseled on the risk of reinfection with HCV after achieving SVR with DAAs. Reinfection is defined as the recurrence of $\mathrm{HCV}$ 
RNA after achieving SVR and demonstration that the infection is caused by a different strain of HCV [4]. In a study reviewing HCV reinfection in co-infected patients 36-75 months after achieving SVR with either Peg-IFN + RBV or Peg-IFN + RBV + DAA, the reinfection rates were estimated to be 8.72 cases per 100 personyears in patients who continued to inject drugs [21]. In Part B of the C-EDGE CO-STAR study evaluating HCV reinfection and injecting risk behavior following grazoprevir/elbasvir therapy in patients receiving OST for at least 3 months, the overall reinfection rate was 2.3 cases per 100 person-years [22]. Data were further extrapolated to show reinfection rates of 4.2 cases per 100 person-years for those who reported continued IDU during the OST treatment period. While this patient population was around 8\% co-infected PWID, further data on reinfection rates in $\mathrm{HIV} / \mathrm{HCV}$ co-infected patients are limited. A meta-analysis showing higher $\mathrm{HCV}$ reinfection incidence in PWID is mostly driven by the HIV-positive MSM cohort, which cannot be extrapolated to the general HIV/HCV population [23]. Patients who become reinfected with HCV should be monitored for spontaneous clearance of infection for at least 3 months prior to restarting therapy [4]. In Part A of the C-EDGE CO-STAR study, 6 out of 301 patients had probable reinfection, and $50 \%$ of these patients had spontaneous clearance of virus [24]. Based on EASL and AASLD-IDSA guidelines, patients who continue to inject drugs should be tested bi-annually or annually for HCV reinfection $[4,8]$. If reinfection is detected, retreatment should be made available to these patients. The combination of sofosbuvir/velpatasvir/voxilaprevir is the regimen of choice for retreatment in patients who failed IFN-free, DAA therapy, although safety and efficacy data on this combination are lacking in the $\mathrm{HCV} /$ HIV co-infected population [4].

The distribution of HCV genotypes/subtypes that previously exhibited specific geographical locations are becoming more prevalent in PWID $[25,26]$. Potential drivers of HCV genotype transmission have been hypothesized and include opiate drug trafficking, increased global travel, and immigration. In the general population. Genotype 1 is the most common genotype seen in North America, accounting for approximately $80 \%$ of reported cases [25]. In PWID, genotype 1a accounts for approximately $70 \%$ of reported cases in North America, followed by genotypes $1 \mathrm{~b}, 2$, and 3 , each occurring at rates of about $10 \%$ [26]. In other geographical areas of the world with a high burden of IDU, genotypes vary in PWID. For example, the most common genotypes in Eastern Europe are $1 \mathrm{a}, 1 \mathrm{~b}$ and 3 , while the most common genotypes in Central Asia are 1a, 1b, 2, 3, and 4. In a study comparing HCV genotypes and subtypes between HIV-positive and HIV-negative patients living in Spain, they found genotype 1 represented $74.6 \%$ of HIV-negative and $58.5 \%$ of HIV-positive patients $(p<0.01)$ [27]. In addition, HCV subtype 1a was the most common in HIV-positive patients (32.1\%) and subtype $1 \mathrm{~b}$ was the most common in HIV-negative patients (53.8\%), while there was an increase in the prevalence of genotype 4 among the HIV/ HCV co-infected population. The increasing distribution of HCV genotypes amongst PWID supports the use of pangenotypic DAAs in this high-risk population when genotypic testing cannot be performed.

Beginning at diagnosis and continuing with each health care encounter, it is important to address and evaluate the patient's illicit drug use. Studies have shown that, in the HIV populations, ART is equally efficacious for PWID when patients are not actively using drugs [28]. Illicit drugs have been linked to depression and anxiety, which is a strong predictor of poor adherence and potentially poor treatment outcomes. Persons living with HIV who inject drugs have lower rates of ART adherence if they have additional risk factors for non-adherence, such as recent incarceration or lack of access to rehabilitation programs. Consequently, management of substance use disorders is often necessary to successfully eradicate $\mathrm{HCV}$ and appropriately manage HIV. Therefore, OST should be considered in combination with DAA therapy when HCV treatment is being suggested for HIV/HCV co-infected patients who continue to inject drugs. In patients living with HIV, OST is associated with a twofold increase in adherence to ART and a $45 \%$ increase in virologic suppression [29]. In patients living with $\mathrm{HCV}$, 
OST does not appear to affect SVR or adherence to DAA therapy $[30,31]$. Most of the DAAs that the FDA approved for use in HIV/HCV co-infected patients are considered safe for co-administration with methadone, buprenorphine, and buprenorphine/naloxone [32-37]. Daclatasvir, ombitasvir/paritaprevir/ritonavir, and ombitasvir/paritaprevir/ritonavir plus dasabuvir increase levels of buprenorphine, requiring increased monitoring for adverse effects without any dose adjustments [38-40]. It is important to build a relationship of trust with these patients, and not only provide medical support for their $\mathrm{HIV} / \mathrm{HCV}$ infections but also assist in connecting them with additional providers to manage comorbidities affecting adherence to medication therapies. This primarily includes a multidisciplinary approach to treating the patients' substance use disorders and psychiatric illnesses. It is prudent to stabilize these patients and provide them with the resources necessary to appropriately manage their HIV and HCV. Further harm-reduction strategies, such as connecting patients with needle exchange programs and/or clinics providing sexually transmitted infection screenings and free condoms, are also important.

There is growing evidence supporting the use of antiviral-based interventions, also known as treatment as prevention (TasP), to reduce transmission of HIV and HCV in PWID [41]. TasP leads to a reduction in the viral burden and infectious potential of patients who continue to engage in high risk behavior. These patients should be counseled on both sexual and injection drug use risk reduction behaviors to avoid transmission and reinfection. Furthermore, patients should be provided with access to naloxone rescue products, and both the patients and family members should be counseled on the signs and symptoms of opioid overdose and appropriate use of naloxone.

Patients enrolled in care for their HIV/HCV can also serve as an access point to enroll other highrisk patients. Respondent-driven sampling (RDS) has become a popular method both in the US and other countries to enroll high-risk patients into studies aiming to treat and prevent HIV and HCV [42-45]. In these studies, "seeds" are identified in high-risk areas and provided with coupons to enroll additional patients for screening and education for HIV and HCV. Similar approaches can be considered in the real-world population by providing patients with educational materials and resources to recruit other PWID into care. At a teaching hospital in Australia, hepatitis B and C screening and treatment was incorporated into the OST setting by a multidisciplinary staff, leading to successful enrollment of high-risk patients into care [46]. By enhancing patient care models in the $\mathrm{HIV} / \mathrm{HCV}$ co-infected population, more patients can receive access to treatment, decreasing the global burden of HCV.

\section{CONCLUSION}

As healthcare providers, the efficacy of $\mathrm{HCV}$ treatment and maintaining HIV virologic suppression are top priorities. Although the PWID population is commonly excluded in $\mathrm{HIV} / \mathrm{HCV}$ co-infection trials, in those that did include this population, successful cure was achieved. These results could therefore be extrapolated to other PWID, especially taking into consideration the large percentage of persons with $\mathrm{HIV} / \mathrm{HCV}$ coinfection being PWID. Arguably, this population makes a good target population for treatment. Novel approaches to providing this treatment should be considered, including meeting these patients in the community. Facilities providing OST, clean needle exchanges, or any type of substance dependence or mental health counseling are key places to engage, link, and retain PWID with HIV/HCV co-infection from the community into care. Furthermore, providing $\mathrm{HCV}$ treatment to PWID can lead to large cost avoidance when taking into consideration the decreased need for liver transplantation, hepatocellular carcinoma and spread of infection through injection drug use. Since many HCV genotypes are prevalent in this patient population, a pangenotypic agent could be optimal first line. Glecaprevir/pibrentasvir is an ideal agent considering the short duration of therapy and limited drug interactions with both HIV and OST therapies. Ultimately taking into account all aspects of care, such as cost, access and efficacy, an interdisciplinary medical team is well 
equipped to provide treatment to prevent HIV and HCV transmission and reduce long-term morbidity in PWID.

\section{ACKNOWLEDGEMENTS}

Funding. No funding or sponsorship was received for this study or publication of this article.

Authorship. All named authors meet the International Committee of Medical Journal Editors (ICMJE) criteria for authorship for this article, take responsibility for the integrity of the work as a whole, and have given their approval for this version to be published.

Disclosures. Rachel M. Murdock, Marisa B. Brizzi, Omar Perez and Melissa E. Badowski have nothing to disclose.

Compliance with ethics guidelines. This article is based on previously conducted studies and does not contain any studies with human participants or animals performed by any of the authors.

Data availability. Data sharing is not applicable to this article as no datasets were generated or analyzed during the current study.

Open Access. This article is distributed under the terms of the Creative Commons Attribution-NonCommercial 4.0 International License (http://creativecommons.org/licenses/ by-nc/4.0/), which permits any noncommercial use, distribution, and reproduction in any medium, provided you give appropriate credit to the original author(s) and the source, provide a link to the Creative Commons license, and indicate if changes were made.

\section{REFERENCES}

1. Degenhardt L, Peacock A, Colledge S, et al. Global prevalence of injecting drug use and sociodemographic characteristics and prevalence of $\mathrm{HIV}, \mathrm{HBV}$, and HCV in people who inject drugs: a multistage systematic review. Lancet Glob Health. 2017;5:e1192-207.

2. Platt L, Easterbrook P, Gower E, et al. Prevalence and burden of HCV coinfection in people living with HIV: a global systematic review and metaanalysis. Lancet Infect Dis. 2016;16(7):797-808.

3. De Angelis D, Sweeting M, Ades AE, Hickman M, Hope V, Ramsay M. An evidence synthesis approach to estimating Hepatitis $\mathrm{C}$ prevalence in England and Wales. Stat Methods Med Res. 2009;18:361-79.

4. EASL. EASL Recommendations on Treatment of Hepatitis C. 2018. http://www.easl.eu/medias/cpg/ 2018/EASL\%20Recommendations\%20on\%20Treat ment $\% 20$ of $\% 20 H e p a t i t i s \% 20 C \% 202018 /$ Englishreport.pdf. Accessed 19 Jul 2018.

5. Tsui JI, Ko SC, Krupitsky E, et al. Insights on the Russian HCV care cascade: minimal HCV treatment for HIV/HCV co-infected PWID in St. Petersburg. Hepatol Med Policy. 2016;1:13.

6. Mehta SH, Lucas GM, Mirel LB, et al. Limited effectiveness of antiviral treatment for hepatitis $\mathrm{C}$ in an urban HIV clinic. AIDS. 2006;20:2361-9.

7. Hagan H, Pouget ER, Des Jarlais DC. A systematic review and meta-analysis of interventions to prevent hepatitis c virus infection in people who inject drugs. J Infect Dis. 2011;204(1):74-83.

8. AASLD-IDSA. Key populations: identification and management of $\mathrm{HCV}$ in people who inject drugs. 2018. https://www.hcvguidelines.org/uniquepopulations/pwid. Accessed 19 Jul 2018.

9. Zeuzem S, Foster GR, Wang S, et al. Glecaprevirpibrentasvir for 8 or 12 weeks in HCV genotype 1 or 3 infection. NEJM. 2018;378(4):354-69.

10. Rockstroh JK, Lacombe K, Viani RM, et al. Efficacy and safety of glecaprevir/pibrentasvir in patients coinfected with hepatitis $\mathrm{C}$ virus and human immunodeficiency virus type 1 : the EXPEDITION-2 study. Clin Infect Dis. 2018;67:1-8.

11. Osinusi A, Townsend K, Kohli A, et al. Virologic response following combined ledipasvir and sofosbuvir administration in patients with HCV genotype 1 and HIV co-infection. JAMA. 2015;313(12):1232-9.

12. Naggie S, Cooper C, Saag M, et al. Ledipasvir and sofosbuvir for HCV in patients coinfected with HIV1. N Engl J Med. 2015;373:705-13. 
13. Wyles D, Brau N, Kottilil S, et al. Sofosbuvir and velpatasvir for the treatment of hepatitis $C$ virus in patients coinfected with human immunodeficiency virus type 1: an open-label, phase 3s. Clin Infect Dis. 2017;65(1):6-12.

14. Rockstroh JK, Nelson M, Katlama C, et al. Efficacy and safety of grazoprevir (MK-5172) and elbasvir (MK-8742) in patients with hepatitis $C$ virus and HIV co-infection (C-EDGE CO-INFECTION): a nonrandomised, open-label trial. Lancet HIV. 2015;2:e319-27.

15. Bentley TS, Phillips SJ, Hanson SG. 2017 US organ and tissue transplant cost estimates and discussion. Milliman Research Report. 2017. http://us. milliman.com/uploadedFiles/insight/2017/2017Transplant-Report.pdf. Accessed 7 Jun 2018.

16. Jinjuvadia R, Salami A, Lenhart A, et al. Hepatocellular carcinoma: a decade of hospitalizations and financial burden in the United States. Am J Med Sci. 2017;354(4):362-9.

17. Cipriano LE, Godlhaber-Fiebert JD. Population health and cost-effectivness implications of a "treat all" recommendation for HCV: a review of the model-based evidence. MDM Policy Pract. 2018. https://doi.org/10.1177/2381468318776634.

18. Iyengar S, Tay-Teo K, Vogler S, et al. Prices, costs, and affordability of new medicines for hepatitis C in 30 countries: an economic analysis. PLoS Med. 2016;13(5):e1002032.

19. Aggarwal R, Chen Q, Goel A, et al. Cost-effectiveness of hepatitis $C$ treatment using generic direct acting antivirals available in India. PLoS ONE. 2017;12(5):e0176503.

20. Gilead Sciences. Gilead subsidiary to launch authorized generics of Epclusa (sofosbuvir/velpatasvir) and Harvoni (ledipasvir/sofosbuvir) for the treatment of chronic hepatitis C. 2018. Available at https://www.gilead.com/news-and-press/press-room/ press-releases/2018/9/gileadsubsidiary-to-launchauth orized-generics-of-epclusa-sofosbuvirvelpatasvir-andharvoniledipasvirsofosbuvir-for-the-treatment-ofchronic.. Accessed 28 Dec 2018.

21. Pineda JA, Nunez-Torres R, Tellez F, et al. Hepatitis $C$ reinfection after sustained virological response in HIV-infected patients with chronic hepatitis C. J Infect. 2015;71(5):571-7.

22. Dore GJ, Grebely J, Altice F, Litwin AH, Dalgard O, Gane $\mathrm{E}$, et al. Hepatitis $\mathrm{C}$ virus reinfection and injecting risk behavior following elbasvir/grazoprevir treatment in participants on opiate agonist therapy: C-EDGE CO-STAR part B. In: AASLD Annual Meeting, October 22nd 2017. Washington, DC. 2017.
23. Simmons B, Saleem J, Hill A, Riley RD, Cooke GS. Risk of late relapse or reinfection with hepatitis $\mathrm{C}$ virus after achieving a sustained virological response: a systematic review and meta-analysis. Clin Infect Dis. 2016;62(6):683-94.

24. Dore GJ, Altice F, Litwin AH, et al. Elbasvir-grazoprevir to treat hepatitis $C$ virus infection in persons receiving opioid therapy. Ann Intern Med. 2016;165(9):625-34.

25. Ruta S, Cernescu C. Injecting drug use: A vector for the introduction of new hepatitis $\mathrm{C}$ virus genotypes. World J Gastroenterol. 2015;21(38): 10811-23.

26. Robaeys G, Bielen R, Ghezel Azar D, Razavi H, Nevens F. Global genotype distribution of hepatitis $\mathrm{C}$ viral infection among people who inject drugs. J Hepatol. 2016;65(6):1094-103.

27. Ramos B, Nunez M, Toro C, et al. Changes in the distribution of hepatitis $\mathrm{C}$ virus (HCV) genotypes over time in Spain according to HIV serostatus: implications for HCV therapy in HCV/HIV-coinfected patients. J Infect. 2007;54(2):173-9.

28. U.S. Department of Health and Human Services. HIV and people who use illicit drugs. Available athttps://aidsinfo.nih.gov/guidelines/html/1/adultand-adolescent-arv/22/hiv-and-people-who-use-illicitdrugs. 2012. Accessed 28 Dec 2018.

29. Low AJ, Mburu G, Welton NJ, et al. Impact of opioid substitution therapy on antiretroviral therapy outcomes: a systematic review and meta-analysis. Clin Infect Dis. 2016;63(8):1094-104.

30. Dore GJ, Altice F, Litwin AH, et al. Elbasvir-grazoprevir to treat hepatitis $C$ virus infection in persons receiving opioid agonist therapy: a randomized trial. Ann Intern Med. 2016;165:625-34.

31. Grebely J, Dore GJ, Zeuzem S, et al. Efficacy and safety of sofosbuvir/velpatasvir in patients with chronic hepatitis $\mathrm{C}$ infection receiving opioid substitution therapy: analysis of phase 3 ASTRAL trials. Clin Infect Dis. 2016;63(11):1479-81.

32. Epclusa $^{\circledR}$ [package insert]. Foster City, CA: Gilead Sciences, Inc. 2016.

33. Harvoni $^{\circledR}$ [package insert]. Foster City, CA: Gilead Sciences, Inc. 2017.

34. Mavyret $^{\circledR}$ [package insert]. North Chicago, IL: AbbVie Inc. 2017.

35. Olysio $^{\circledR}$ [package insert]. Titusville, NJ: Janssen Therapeutics. 2013. 
36. Solvadi ${ }^{\circledR}$ [package insert]. Foster City, CA: Gilead Sciences, Inc. 2017.

37. Zepatier ${ }^{\circledR}$ [package insert]. Whitehouse Station, NJ: Merck \& Co., Inc. 2018.

38. Technivie ${ }^{\circledR}$ [package insert]. North Chicago, IL: AbbVie Inc. 2017.

39. Viekira $\mathrm{XR}^{\circledR}$ [package insert]. North Chicago, IL: AbbVie Inc. 2017.

40. Daklinza ${ }^{\circledR}$ [package insert]. Princeton, NJ: BristolMyers Squibb Company. 2017.

41. Coffin PO, Rowe C, Santos GM. Novel interventions to prevent HIV and HCV among persons who inject drugs. Curr HIV/AIDS Rep. 2015;12:145-63.

42. Neaigus A, Reilly KH, Jenness SM, et al. Trends in HIV and HCV risk behaviors and prevalent infection among people who inject drugs in New York City. J Acquir Immune Defic Syndr. 2017;75:S325-32.
43. Abadie R, Welch-Lazoritz M, Gelpi-Acosta C, Reyes JC, Dombrowski K. Understanding differences in $\mathrm{HIV} / \mathrm{HCV}$ prevalence according to differentiated risk behaviors in a sample of PWID in rural Puerto Rico. Harm Reduct J. 2016;13:10.

44. Solomon SS, McFail AM, Lucas GM, et al. Respondent-driven sampling for identification of HIV-and $\mathrm{HCV}$-infected people who inject drugs and men who have sex with men in India: a cross-sectional, community-based analysis. PLOS Med. 2017;14(11):e1002460.

45. Wenz B, Nielsen S, Gassowski M, et al. High variability of HIV and HCV seroprevalence and risk behaviours among people who inject drugs: results from cross-sectional study using respondent-driven sampling in eight German cities (2011-14). BMC Public Health. 2016;16:927.

46. Weltman M, Fragomeli V. Addressing viral hepatitis in the opiate substitution setting: an integrated nursing model of care. J Gastroentrol Hepatol. 2015;30(Suppl. 2):6-11. 